evolution. But otherwise I think it is an exciting concept that will do much to stimulate debate and enliven thinking in the usually staid field of mineralogy.

Minik T. Rosing is at the Nordic Center for Earth Evolution, Natural History Museum of Denmark, University of Copenhagen,
DK 1350 Copenhagen, Denmark.

e-mail:minik@snm.ku.dk

1. Johnsen, O. Minerals of the World (Princeton Univ. Press, 2002).

2. Hazen, R. M. et al. Am. Mineral. 93, 1693-1720 (2008).

3. Bowen, N. L. The Evolution of the Igneous Rocks (Princeton Univ. Press, 1928)

\title{
PHOTONICS
}

\section{Nanomechanics gets the shakes}

\author{
Tobias J. Kippenberg
}

\section{Photonic circuits can allow light to be tightly confined on a chip. A clever experiment reveals how this process can be exploited to create optical forces that drive a nanoscale mechanical oscillator.}

In the 1970s, Arthur Ashkin at Bell Laboratories showed ${ }^{1}$ that the force that light exerts on an object - the radiation pressure force can be used to trap particles. This discovery has led to a revolution in the use of lasers to trap objects ranging from atoms to biological cells. With 'optical tweezers', for example, a laser beam is focused tightly on the surface of an object, and a spatial variation in the optical intensity creates a gradient force that causes the object to be attracted to the centre of the beam, where the intensity is highest. On page 480 of this issue, Li et al. ${ }^{2}$ describe how they have used nanoscale photonic waveguides on a silicon chip to exert optical gradient forces on a nanomechanical oscillator - a first step towards unifying nanophotonics and nanomechanics.

Observing how the radiation pressure force can affect a mechanical oscillator has been an experimental challenge for decades, although the use of nano- and micromechanical elements has allowed this weak force to be observed. For example, by using cavity optomechanical systems, in which photons are tightly coupled to the motion of an oscillator, both amplification and 'cooling' (the opposite of amplification) of the mechanical motion have been achieved ${ }^{3}$. But so far, researchers have observed only the scattering force that results when momentum is transferred as the light is reflected directly, and this force is parallel to the direction of light propagation. Gradient forces, in contrast, are transverse to the light beam. It has been noted ${ }^{4}$ that gradient forces might occur in integrated photonic circuits, in which waveguides of a scale similar to the light's wavelength tightly confine the light. But these forces, which could be applied on a chip, haven't yet been observed.

Li et al. ${ }^{2}$ use a clever design in which a single device constitutes both an optical waveguide and a nanomechanical resonator. Integration of the resonator is seamlessly accomplished by sending light through a nanophotonic waveguide, a portion of which is suspended to form a nanomechanical beam. This 'optomechanical' waveguide thereby serves both to confine light and to act as a mechanically compliant element that can vibrate at its fundamental frequency -10 megahertz in the present study.

Crucial requirements for observing gradient forces are that the waveguides are so small that part of the optical field is evanescent (decaying exponentially with distance) and extends outside the waveguide into the substrate. The latter requirement is achieved by suspending the nanophotonic waveguide very close to a non-conducting (dielectric) substrate. In Li and colleagues' experiment, the gap between the waveguide and the (oxide) substrate is only 300 nanometres (about a fifth of the wavelength of the light used). A periodically varying gradient force can then be obtained by subjecting the light beam to a modulated power. Tuning this modulation to the resonant frequency of the nanomechanical resonator drives the oscillation of the nanomechanical beam.

Driving mechanical beams is not a new idea. What is new is exciting their motion using optical gradient forces, as this should allow a much larger bandwidth than other methods, possibly extending into the microwave regime ${ }^{5,6}$. Moreover, the entire actuation method can take place 'on a chip' and does not require external fields as do other methods of actuation, such as magnetomotive forces.

But how can we be sure that the gradient force really is the mechanism responsible for the oscillations? Detecting radiation pressures has long eluded researchers. One other factor known to trigger such oscillations ${ }^{7,8}$ is the thermal effect resulting from thermal expansion, or heating of residual background gas molecules; these can mimic the effects of radiation pressure on the nanomechanical beam. A light mill, which can be bought from any toy store, is an example of a device that rotates through thermal effects rather than through radiation pressure .
Li et al. ${ }^{2}$ set out to quantify thermal effects on the nanomechanical beam. Their experiments, performed in a high vacuum, reveal counterintuitively that, despite the nanoscale dimension of the waveguide, thermal gradients build up over a time period that is much longer than the period of the mechanical oscillator. Therefore, by using a sufficiently fast modulated light beam, the authors could be sure that most of the resulting periodically varying force was due to the optical field gradient rather than to thermal effects. At gigahertz frequencies, thermal effects could in fact be eliminated entirely.

The observation of gradient optical forces on a silicon chip has wide-ranging implications for the field of nanophotonics. Li and colleagues' work demonstrates that these forces can be exploited in nanophotonic circuits to trigger nanomechanical oscillations. But the principle of their experiment points to more general ways of incorporating nanomechanical and nanophotonic elements on a silicon chip. Silicon's useful mechanical properties (such as its low mechanical dissipation) have already been used to create the most sensitive mechanical beams to date ${ }^{10}$. But Li and colleagues' work points to a whole new class of photonic functionality - the use of gradient forces - in optically driven mechanical oscillators, photonic mixers ${ }^{11}$ and narrow-band radio-frequency filters. As such, gradient optical forces might become a new element in 'silicon photonics'.

Probably the greatest remaining challenge is extending the method to higher resonant frequencies, which will require an increase in the optical power needed to produce the mechanical oscillations. In the authors' experiment this power is already quite high - on the order of tens of milliwatts. One solution would be to reduce the gap between the waveguide and the substrate. Alternatively, the use of cavity nano-optomechanical systems, such as integrated silicon microresonators, would not only decrease the power required by orders of magnitude, but would also provide a route to drive the mechanical oscillator without time-varying optical fields ${ }^{3}$.

Tobias J. Kippenberg is at the Ecole Polytechnique Fédérale de Lausanne (EPFL), 1015 Lausanne, Switzerland, and is partially affiliated with the Max-Planck-Institut für Quantenoptik, Garching, Germany.

e-mail: tobias.kippenberg@epfl.ch

\footnotetext{
Ashkin, A. Phys. Rev. Lett. 24, 156-159 (1970).

2. Li, M. et al. Nature 456, 480-484 (2008).

3. Kippenberg, T. J. \& Vahala, K. J. Science 321, 1172-1176 (2008).

4. Povinelli, M. L. et al. Opt. Lett. 30, 3042-3044 (2005)

5. Huang, X. M. H., Zorman, C. A., Mehregany, M. \& Roukes, M. L. Nature 421,496 (2003)

6. Carmon, T. \& Vahala, K. J. Phys. Rev. Lett. 98, 223902 (2007).

7. Metzger, C. H. \& Karrai, K. Nature 432, 1002-1005 (2004)

8. Zalalutdinov, M. et al. Appl. Phys. Lett. 79, 695-697 (2001)

9. Woodruff, A. E. Physics Teacher 6, 358-363 (1968).

10. Rugar, D., Budakian, R., Mamin, H. J. \& Chui, B. W. Nature 430, 329-332 (2004).

11. Hossein-Zadeh, M. \& Vahala, K. J. Phot. Tech. Lett. 19, 1045-1047 (2007).
} 